\title{
CFD ANALYSIS OF THE SPACER GRIDS AND MIXING VANES EFFECT ON THE FLOW IN A CHOSEN PART OF THE TVSA-T FUEL ASSEMBLY
}

\author{
JAKUB JUKLÍČEK ${ }^{a, *}$, VÁCLAV ŽELEZnÝ ${ }^{b}$ \\ ${ }^{a}$ Czech Technical University in Prague, Technická 4, Praha 6, 166 07, Czech Republic \\ ${ }^{b}$ Power Engineering Department, Czech Technical University in Prague, Technická 4, Praha 6, 166 07, Czech \\ Republic \\ * corresponding author: jakub.juklicek@gmail.com
}

Abstract. CFD is a promising and widely spread tool for flow simulation in nuclear reactor fuel assemblies. One of the limiting factors is the complicated geometry of spacer grids. It leads to a computational mesh with a high number of cells and with a possibility of decreasing quality. An approach which simulates the flow as precisely as possible and simultaneously at a reasonable computational expense therefore has to be chosen. The goal of the following CFD analysis is to obtain a detailed velocity field in a precise geometry of a chosen part of the TVSA-T fuel assembly. This kind of simulation provides data for comparison that can be applied in many situations, for instance, for comparison with simulations when a porous media boundary condition is applied as a replacement for the spacer grid.

The TVSA-T fuel assembly is equipped with combined spacer grids. A combined spacer grid has two functions. The first function is to support the fuel pins as a part of the assembly skeleton. The second function is to ensure coolant mixing with the mixing vanes. The support part is geometrically very complicated. Therefore it is impossible to prepare a good quality computational mesh there. It is also difficult to create a mesh in the support part and the mixing part joint area because of the inaccurate connection between these two parts.

A representative part of the TVSA-T fuel assembly with a combined spacer grid segment was chosen to perform the CFD simulation. Some inevitable simplifications of the spacer grid geometry were performed. These simplifications were as insignificant as possible to preserve the flow character and to make it possible to prepare a quality mesh at the same time.

A steady state CFD simulation was performed with the $k-\varepsilon$ realizable turbulence model. Heat transfer was not simulated and only the velocity field was investigated. The detailed flow characterization which was obtained from this calculation has shown that mixing vanes already affect the flow in the support part of the grid thanks to the suction effect. The vortex structures disappear approximately $50 \mathrm{~mm}$ behind the mixing vanes but the basic spiral character of the flow is preserved in the whole area between two following spacer grids.

KEYWORDS: TVSA-T fuel assembly; spacer grid; mixing vanes; CFD; turbulent flow.

\section{INTRODUCTION}

Thermalhydraulic analysis is needed to predict the flow and temperature distributions in fuel assemblies to ensure the safe operation of nuclear reactors. Analysis of this kind can be performed by commercial CFD codes. A limiting factor in these calculations is the large computational domain of the fuel assembly which leads to unrealistic computational time or to a large computational mesh which is not computable with current hardware options available in most research facilities. The geometrically most complicated parts are the inlet of the fuel assembly, the spacer grids and the fuel assembly outlet. These parts have to be simplified to create a computational geometry where calculation is feasible.

TVSA-T fuel is currently used in the Temelin NPP. The goal of this work is to simulate the flow in a chosen part of the TVSA-T fuel assembly with a spacer grid segment. The TVSA-T spacer grid has two functions: support of the assembly skeleton and coolant mixing. The spacer grid is therefore divided into two connected parts - the support part and the mixing vanes. Both parts had to be simplified. The connection between these parts was also simplified and a computational mesh was successfully created. A steady state CFD simulation was performed to examine the velocity field. The velocity field was evaluated to describe the spacer grid effect on the flow character in the fuel assembly.

\section{TVSA-T SPACER GRID GEOMETRY}

The support part is made of cells which provide support for the fuel pin in three points. The mixing vanes are placed on the top edge of metal strips forming the mixing part of the spacer grid. Both parts are 

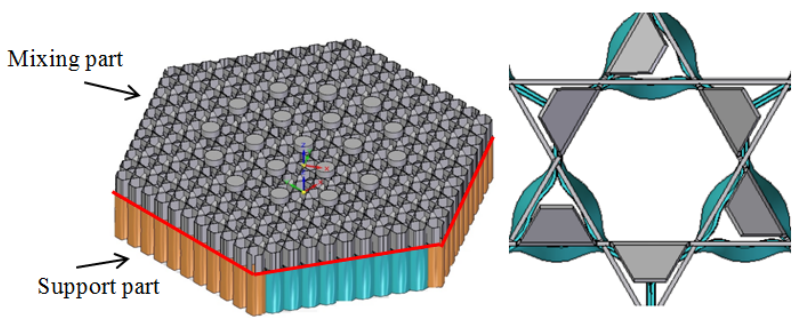

Figure 1. TVSA-T spacer grid with the detail of the support/mixing part transition.

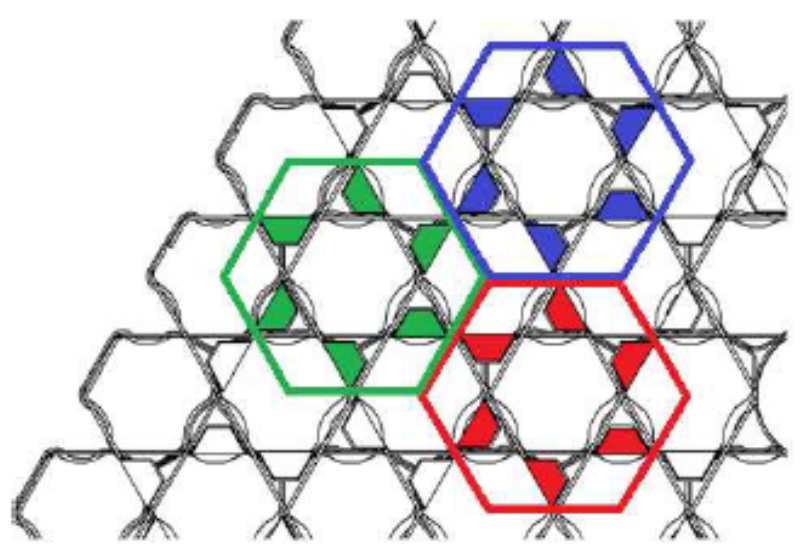

Figure 2. Symmetrical geometry of the mixing part.

welded together and form a complete TVSA-T spacer grid, as illustrated in Fig. 11 The red line indicates the transition between those two parts. The support part had to be simplified, for example, in places where the grid touches the fuel cladding. Here some problematic radiuses were replaced by straight edges to prevent high skewness of the cells. A simplification was also performed in the transition area between the two parts of the grid. The mixing part directly follows the simplified support part. Slight modifications were done also in the mixing vanes geometry.

\subsection{ORIGINAL GEOMETRY}

The original geometry of the mixing part consists of a symmetrical configuration of the mixing vanes. The mixing vanes are directed around the fuel pin to create the spiral character of the coolant flow. This configuration is illustrated in Fig. 2, where the identification by different colours shows that the configuration is periodically repeated every 120 degrees. Fig. 3 illustrates the transition between the support part (yellow) and the mixing part (red). This transition is indicated by the red line in Fig. 1, as mentioned before. The blue colour indicates a small cross section area that has been simplified.

\subsection{Simplified GeOMETRY}

The comparison of the original and simplified geometry of the support cell is illustrated in Fig. 4 The radiuses were replaced by straight edges. The support point was preserved as the connection point between the fuel cladding and the support cell. This provision helped

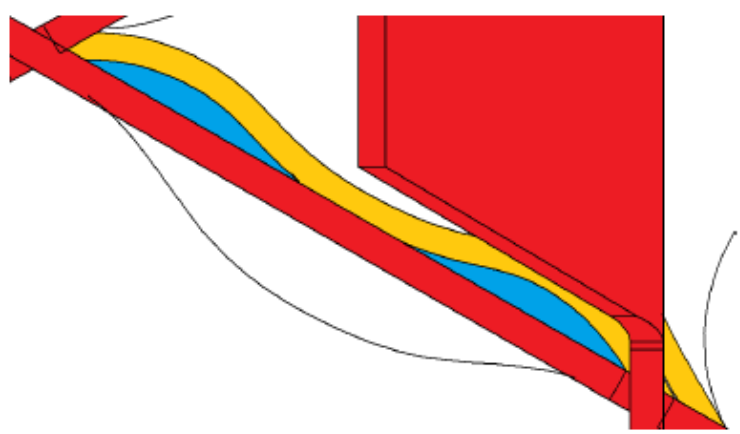

FiguRE 3. Details in the transition between the support and mixing part.
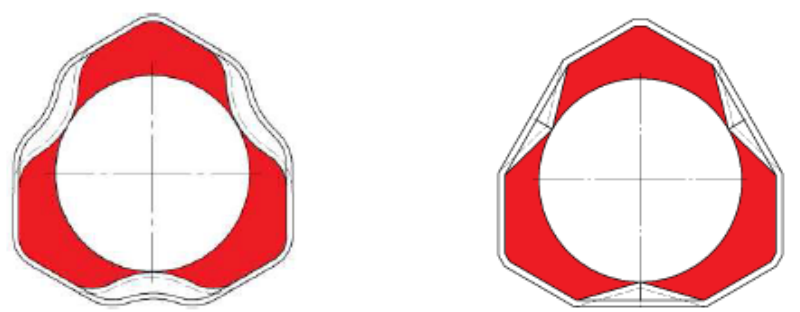

Figure 4. Original (left) and simplified (right) geometry of the support cell in the fuel pin support plane.

to enlarge the angle between the fuel pin and the support cell, which consequently enabled the creation of the computational mesh in this part of the grid. The support point was the most complicated part in terms of meshing.

Fig. 5illustrates the final computational geometry used for CFD analysis. The support part and the mixing part are directly connected (the small flow cross sections illustrated in Fig. 3 are not modelled). The arrows indicate the use of the periodic boundary condition to explain the already mentioned 120 degrees symmetry. The volumes for the flow development in front of and behind the spacer grid are also part of the computational geometry. These volumes are more widely described in the following section.

\section{CFD ANALYSIS}

\subsection{Computational mesh}

The computational mesh was created using the Gambit 2.4.6 software. The computational geometry was divided into three main parts with a transition volume between each part: the volume for the flow development before the spacer grid $(200 \mathrm{~mm})$, the spacer grid area and the volume for the flow development behind the spacer grid $(500 \mathrm{~mm})$. The distance recommended to develop the flow (20 hydraulic diameters) is of $200 \mathrm{~mm}$ and the distance between two following spacer grids is of $500 \mathrm{~mm}$. The mesh in those volumes is illustrated in Fig. 6. Moreover, the computational mesh in the spacer grid area is divided into two parts: the support part and the mixing vanes part. This mesh division is just the logical approach to create the mesh, 

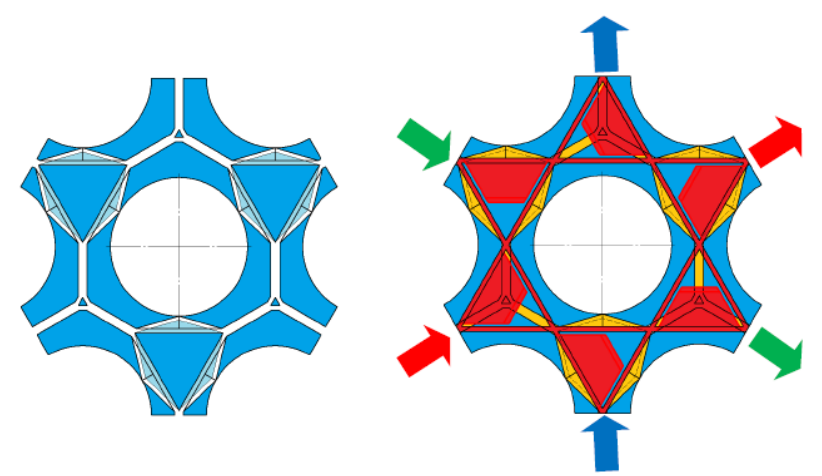

FIGURE 5. Final simplified computational geometry.

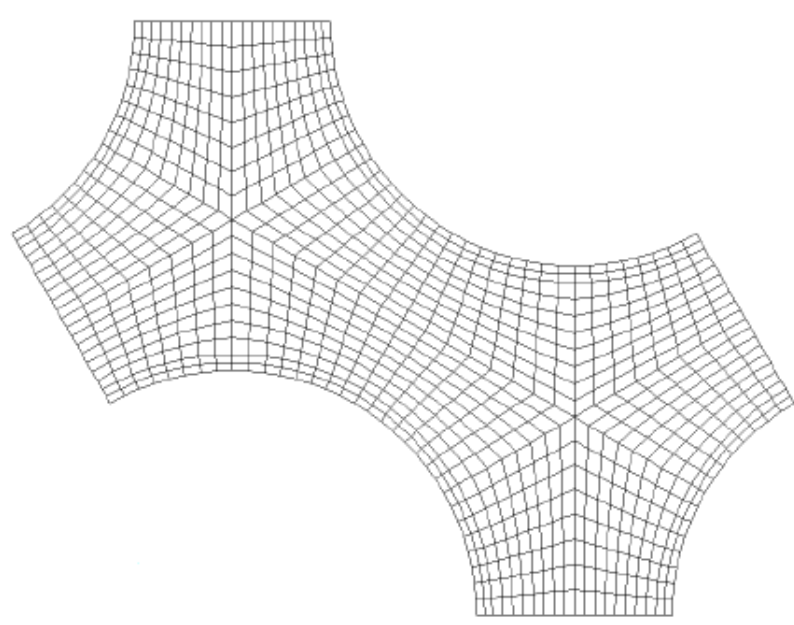

Figure 6. Mesh in the volumes for the flow development.

and no interfaces are present in the geometry. The mesh consists of 5593797 cells. The most problematic part is the support cell area around the support point with 2421032 cells ( $43 \%$ of the cells), as illustrated in Fig. 7. This area was meshed with tetrahedrons and the maximum EquiSize Skew is 0.95. The high EquiSize Skew did not affect the solution convergence. The transition volumes and the volumes in the mixing vanes area were also meshed with tetrahedrons. The other parts were meshed with hexahedral or wedge cells. The mesh in the volumes to develop the flow is structured.

\subsection{BOUNDARY CONDITIONS}

The boundary conditions correspond to the operational conditions of the VVER-1000 nuclear reactor in the Temelin NPP [1]. The operational pressure is 15.7 MPa and the temperature is $290^{\circ} \mathrm{C}$. The water properties were set according to the following parameters: density $\varrho=746.5 \mathrm{~kg} / \mathrm{m}^{3}$ and dynamic viscosity $\eta=9.25 \cdot 10^{-5} \mathrm{Pas}$. The velocity inlet boundary condition was set at the inlet with velocity $v=5.536 \mathrm{~m} / \mathrm{s}$. The hydraulic diameter at the inlet $d_{H}=0.0106 \mathrm{~mm}$. The pressure outlet boundary condition was set at the outlet. The periodic boundary condition was set as shown before in Fig. 5 .

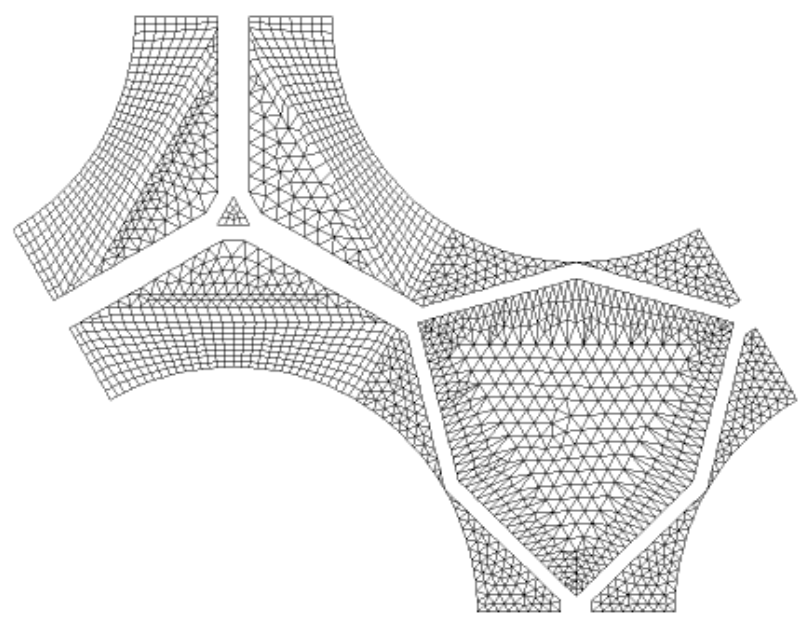

Figure 7 . Mesh in the fuel pin support plane.

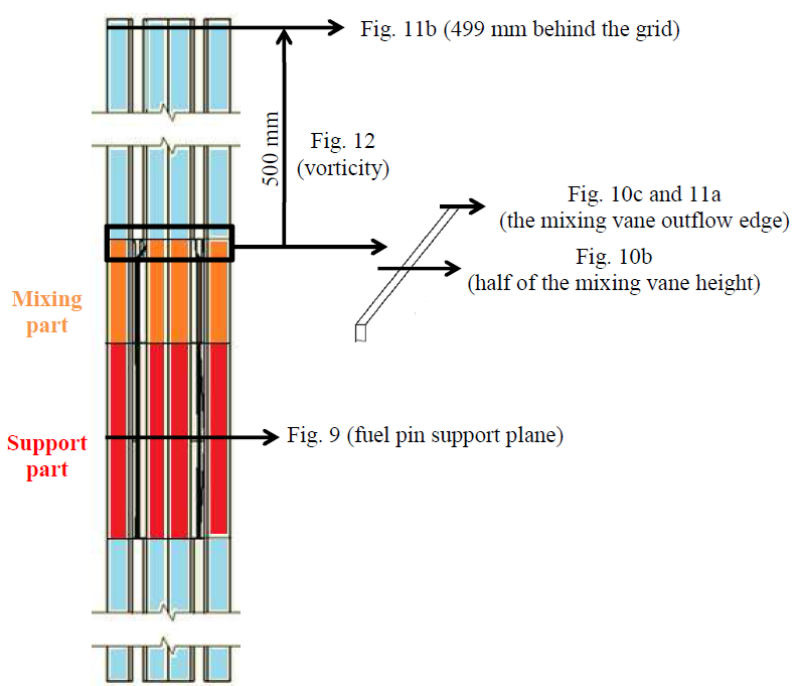

Figure 8. Results location.

\subsection{Models And CAlCUlation SETtings}

The CFD simulation was performed using the ANSYS FLUENT 14.5 CFD code. The $k-\varepsilon$ realizable turbulence model was used in the calculation. The turbulence intensity $I=3 \%$ was set according to the Reynolds number $\mathrm{Re}=473576$ [2]. The range of the dimensionless wall distance $y^{+}$was $30<y^{+}<300$. The solution has converged for steady state simulation. Second order upwind spatial discretization was used. Heat transfer was not simulated.

\section{Results}

Fig. 8 illustrates the location of the following cross sections, where some of the results are shown. The blue colour indicates the volumes to develop the flow in front of the spacer grid and behind it.

The velocity field obtained from the calculation was analysed and the spacer grid influence on the coolant flow was evaluated. Fig. 9 illustrates the velocity field in the plane with the support point of the fuel pin (axial velocity contours). Wall functions are not applicable in the cells closest to the support point. 


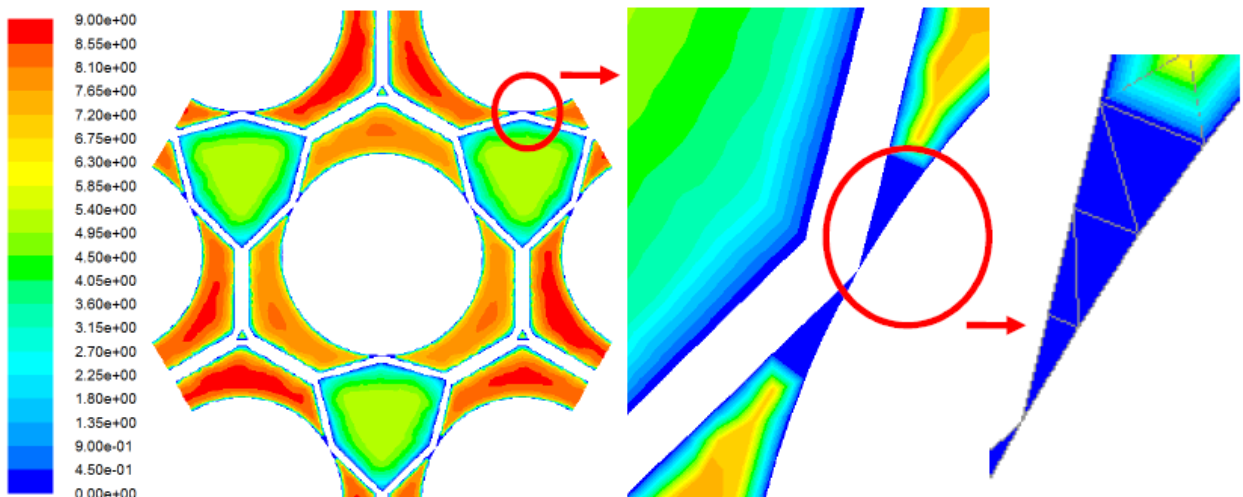

Figure 9. Contours of axial velocity in the support point plane $[\mathrm{m} / \mathrm{s}]$.

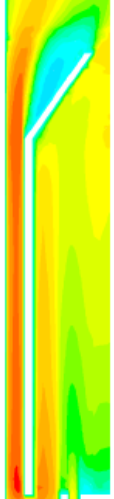

a

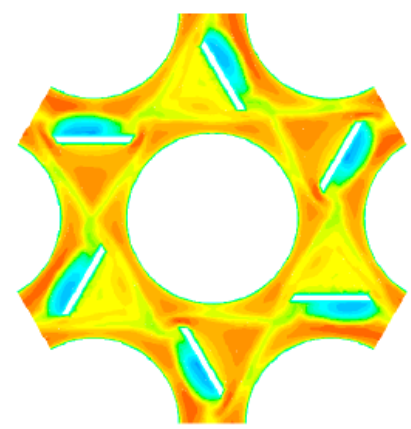

$\mathrm{b}$

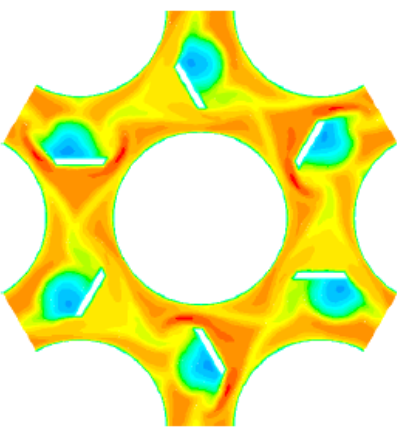

Figure 10. Contours of axial velocity in the mixing vanes area $[\mathrm{m} / \mathrm{s}]$.

Therefore, the velocity field is not evaluated in these cells, and more significant simplifications could be done in this area without affecting the flow character. Simplifying this detail would also be beneficial in terms of mesh quality and, subsequently, of lowering the number of mesh cells. In the next paragraph the suction effect of the mixing vanes is described. This effect is also noticeable in Fig. 9 as the area with the highest coolant speed.

Fig. 10 illustrates the contours of axial velocity in the mixing vanes area. It can be seen that the mixing vanes already affect the flow in the support part of the grid (Fig. 10p). The sudden widening of the flow crosssection (located behind the mixing vanes) causes the suction effect, which leads to coolant acceleration in the support part. Cross-sections in the mixing vanes area show the formation of the vortex structures as indicated also in Fig. 10p and Fig. 10k. These vortex structures are formed alongside the entire mixing vane. The vortex structures are also directed opposite to the axial flow, which explains the negative values of the velocity.

Fig. 11 illustrates the velocity vectors in the cross flow direction. The vortex structures are illustrated in Fig. 11a. These vortex structures disappear approximately $50 \mathrm{~mm}$ behind the spacer grid, as can be seen in Fig. 12, where the average vorticity in horizontal cross sections is illustrated. The $0 \mathrm{~mm}$ level in Fig. 12 corresponds to the outflow edge of the mixing vanes, and $500 \mathrm{~mm}$ is the distance between the two following spacer grids, as was already mentioned. The vorticity remains constant after the $50 \mathrm{~mm}$ level and further behind the spacer grid. Fig. $11 \mathrm{~b}$ illustrates that the spiral character of the flow is preserved in the whole volume behind the spacer grid. The velocity vectors in Fig. 11p illustrate the plane just before the following spacer grid ( $499 \mathrm{~mm}$ from the mixing vanes outflow edge). Axial flow is already strongly dominant in this area but the arrows indicate that the flow character is still affected by the effect of the mixing vanes.

\section{Conclusions}

A spacer grid affected velocity field in the TVSA-T fuel assembly was obtained in the CFD simulation after the original geometry was successfully simplified and meshed. Only necessary simplifications without any major changes were made in the grid geometry to mesh the geometry of the spacer grid qualitatively. It was shown that precise modelling is feasible in terms of meshing, but a large number of cells is consequently reached. The support point between the fuel pin and the support cell was preserved, but a finer mesh with a lower number of cells could be achieved through a greater simplification without significantly affecting 


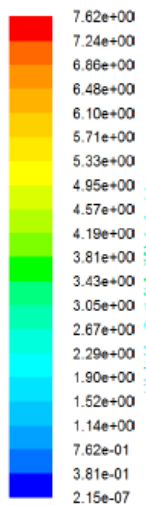

$-15 e-07$

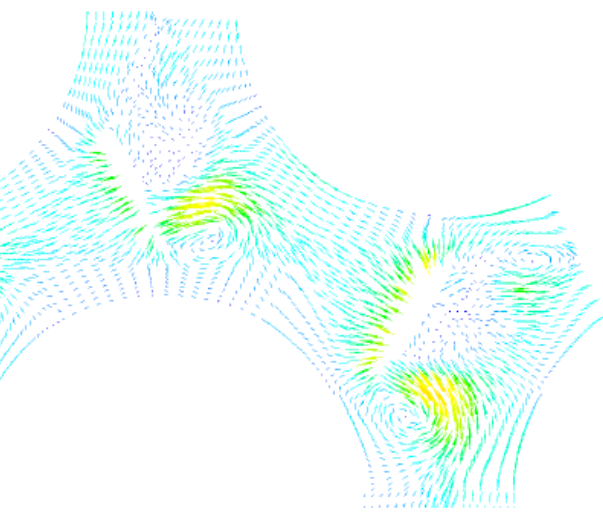

a

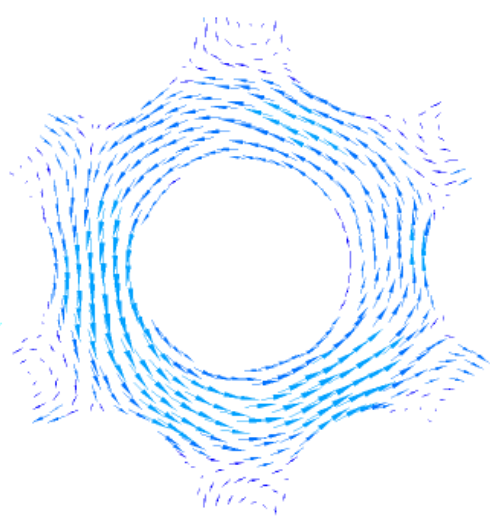

$\mathrm{b}$

FiguRE 11. Vectors of cross-flow velocity $[\mathrm{m} / \mathrm{s}]$.

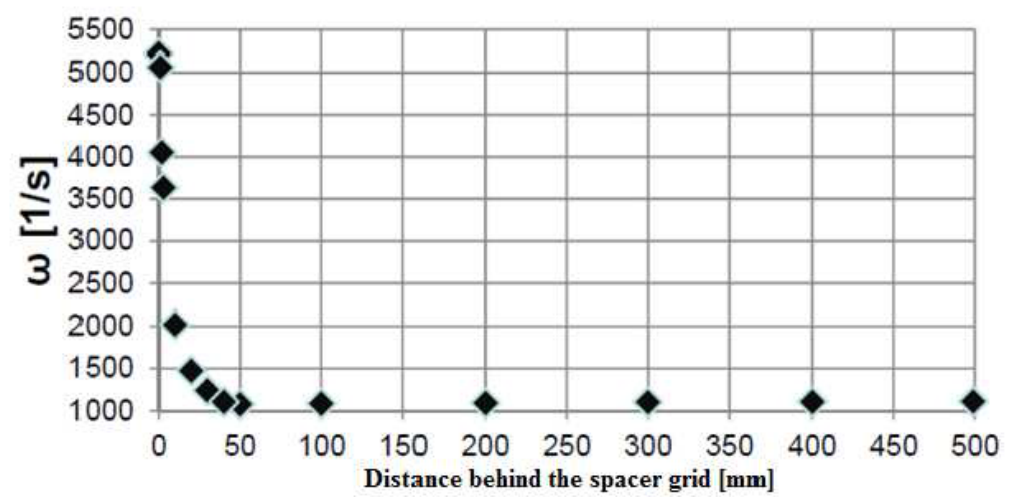

Figure 12. Average vorticity $\omega$ in horizontal cross sections behind the spacer grid.

the flow character. CFD analysis showed that the spiral character of the flow is preserved in the whole volume between the two following spacer grids. Simulation had also shown that mixing vanes already affect the flow in the support part of the grid, thanks to the suction effect. Mixing vanes form vortex structures alongside their geometry. These vortex structures disappear approximately $50 \mathrm{~mm}$ behind the spacer grid. It should be stated that this simulation does not correspond to the real flow conditions in the TVSA-T fuel assembly (e.g., flow affected by the fuel assembly inlet or by the previous spacer grid). Also, if a larger area (more fuel pins) is simulated, the spiral character of the flow is limited and the cross-flow between the subchannels takes on a more significant role (this occurs approximately $100 \mathrm{~mm}$ and further behind the spacer grid) 3. Nevertheless, this kind of simulation provides comparative data for more significant simplifications of the fuel assembly geometry or for replacement of the part or whole spacer grid geometry by the porous media boundary condition in CFD simulation. Computational mesh could also serve as input for calculations of larger areas with more fuel pins and, e.g., two spacer grids if appropriate hardware and software is available in the research or industrial facility.

\section{ACKNowledGements}

This work was done as partial fulfilment of the requirements for the degree of Master of Science in Nuclear Power Devices at the Czech Technical University in Prague, Faculty of Mechanical Engineering.

\section{REFERENCES}

[1] Temelín, hlavní technické údaje, $2013 .$. http://www.cez.cz/cs/vyroba-elektriny/
jaderna-energetika/jaderne-elektrarnycez/ete/
technologie-a-zabezpeceni/2.html [2015-03-20]

[2] Ansys Fluent 12.0 User's Guide. ANSYS, Inc. 2009.

[3] Železný, V., Zácha, P. Úvodní simulace proudění skrze oblast distančních mřížek a turbulizujících lopatek ve vybrané skupině palivových kanálů souboru TVSA-T. Řež, 2011. 